\title{
Does eating a reheated starchy carbohydrate meal improve postprandial glycaemia?
}

\section{Abstract}

Increased postprandial glycaemia and reduced insulin sensitivity are associated with development of Type 2 Diabetes (T2D). Maintaining a normal glucose response is important both for healthy individuals, for disease prevention, and for those with T2D, to prevent development of diabetes-related complications. Chilling previously-cooked starchy carbohydrate (CHO) results in retrogradation of some of the starch to form resistant starch (RS). RS is not absorbed in the small intestine and consequently does not contribute to the postprandial glucose excursion. Reheating the $\mathrm{CHO}$, however, reverses this process, reducing some of the RS content. RS type 5 is formed in the laboratory by heating starch with free fatty acids; there is limited evidence for its formation using domestic cooking methods and real foods. Furthermore it is unclear whether this would translate into a noticeable effect on postprandial glucose metabolism.

In this randomised crossover study, 8 participants attended two study days; at one they consumed a freshly cooked mashed potato meal ( $203 \mathrm{~g}$ boiled potato, $25 \mathrm{~g}$ butter) at the other they consumed an identical meal which had been chilled for $66 \mathrm{~h}$ then microwave reheated. The potatoes were intrinsically labelled with $\left[\mathrm{U}_{-}^{13} \mathrm{C}\right] \mathrm{starch}$, and participants received a variable $\left[6,6-{ }^{2} \mathrm{H}_{2}\right] \mathrm{glucose}$ infusion, allowing detailed glucose flux modelling. Venous blood samples were taken for $6 \mathrm{~h}$ postprandially.

There was no significant effect on postprandial glucose, however repeated measures ANOVA on postprandial insulin time-point data found a significant difference between meals $(\mathrm{p}=0.026)$, with a $24 \%$ reduction in incremental area under the curve $(0-120$ $\min$ ) and $21 \%$ reduction in insulin peak between freshly cooked and reheated meals. There were no significant effects on rate of appearance of glucose into the plasma from the gut $\left(\mathrm{R}_{\mathrm{a}}\right)$ or on endogenous (hepatic) glucose production (EGP), however there was a strong trend for a reduced rate of glucose disposal (uptake into tissues, $R d)$ following the reheated meal $(p=0.054)$.

It is hypothesised that RS was formed in the reheated meal by the chilling and reheating process; this will be verified by in vitro work later in the project. The attenuation of the postprandial insulin response with no significant effect on EGP suggests enhanced hepatic insulin sensitivity following the reheated meal as a possible mechanism for the effects of RS on postprandial glycaemia. This study demonstrates that making simple changes to the way a starchy carbohydrate meal is prepared can have significant beneficial effects on postprandial glucose metabolism.

\section{Conflict of Interest}

There is no conflict of interest 